\title{
Edición de tablas de muestreo
}

\author{
Sampling tables edition \\ Jorge Ortiz Pinilla \\ jorgeortiz@usantotomas.edu.co
}

\begin{abstract}
Resumen
Se propone un procedimiento y una función en lenguaje $\mathrm{R}$ para preparar la edición de grandes cantidades de tablas con estructuras similares que se generan durante las aplicaciones de encuestas.
\end{abstract}

Palabras clave: $\mathrm{AT}_{\mathrm{E}} \mathrm{X}$, estudios por muestreo, estimación, elaboración de informes de muestreo.

\begin{abstract}
We propose a methodology and a function in $\mathrm{R}$ language to prepare the edition of a great volume of tables with similar structures comming from the application of sampling studies.
\end{abstract}

Key words: $\mathrm{AT}_{\mathrm{E}} \mathrm{X}$, sampling studies, sampling report generation.

\section{Introducción}

Algunos programas estadísticos comerciales ofrecen módulos para elaborar tablas complejas con una presentación muy cercana de la que se necesita para los informes de los trabajos de investigación. Sin embargo, quienes lo han vivido saben que para grandes cantidades de tablas, la complejidad del código hace que el trabajo de edición sea dispendioso y confuso. En una publicación anterior, Ortiz Pinilla \& Bautista (2006) presentamos una propuesta para el manejo de tablas en IATEX con SAS/SQL.

Con el presente artículo generalizamos esa propuesta con una función más flexible que incluye la posibilidad de variar la cantidad de columnas de las tablas sin necesidad de modificar el código. El cambio al lenguaje $\mathrm{R}$ se debe a que se trata de un software libre, con versiones en varios sistemas operativos y con la posibilidad de integrarse con programas de amplio uso como SPSS en sus versiones más recientes.

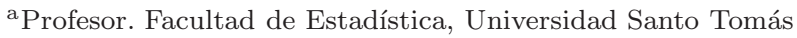




\section{Método}

Una vez definido el formulario de recolección de información y a partir de los objetivos planteados, el investigador concibe un conjunto de tablas que serán la base de sus análisis y que constituirán una parte esencial de su informe. Ya desde este momento puede iniciar la preparación de la edición, de manera que cuando se recojan los datos, los tiempos de este proceso, generalmente muy dispendioso, se reduzcan y dejen un espacio para el análisis mismo de los resultados.

La preparación consiste básicamente en identificar los elementos que componen una tabla y agruparlos en objetos diferentes para tenerlos disponibles en el momento de construirla. En la que se presenta a continuación se los destaca con números resaltados entre paréntesis:

Tabla 1: Título de la tabla (1)

\begin{tabular}{|c|c|c|c|c|}
\hline \multirow{3}{*}{$\begin{array}{l}\text { Encabezado para las } \\
\text { filas (3) }\end{array}$} & \multirow{3}{*}{$\begin{array}{r}\text { Total po- } \\
\text { blacional } \\
(4)\end{array}$} & \multicolumn{3}{|c|}{ Encabezado para las columnas (2) } \\
\hline & & \multicolumn{3}{|c|}{ Subpoblación Subpoblación Subpoblación } \\
\hline & & $\mathrm{A}(5)$ & B (5) & $\mathrm{C}(5)$ \\
\hline Campo/variable 1 (6) & $x_{11}$ & 14.8 & $x_{13}$ & 19.8 \\
\hline$($ cve \%) & 2.1 & 1 & 3.4 & 3.7 \\
\hline Campo/variable 2 (6) & Total (7) & 4.8 & Datos (8) & 5.7 \\
\hline$($ cve \%) & 4.1 & 2.1 & 1.6 & 2.4 \\
\hline
\end{tabular}

Nota de tabla (9)

Cada tabla es una disposición rectangular de los valores de las estimaciones (8) obtenidas para varios grupos poblacionales (5) de un conjunto de campos del formulario u otras variables que se calculan en función de ellos (6). Se incluye una estimación para el total de la población estudiada (7). Para la descripción de la tabla se necesita un título (1), un encabezado para las columnas (2), que se obtiene del nombre de la variable categórica que determina los grupos poblacionales, un encabezado para las filas (3), que describe el tipo de información que se está resumiendo en la tabla, un descriptor para la columna de totales (4) y eventualmente una nota con alguna explicación que se considera importante (9).

En las secciones siguientes, presentadas en el orden sugerido para organizar sin inconvenientes la preparación de la edición de las tablas, se describe en detalle cada uno de estos elementos.

\subsection{Rótulos de las variables categóricas}

En el formulario de recolección de información se buscan los campos que corresponden a variables categóricas y se identifican las categorías de respuesta. El formulario asigna a cada categoría un código numérico para identificarla y un rótulo 
que la describe. Un conjunto de parejas de códigos y rótulos se llama un tipo de rótulo y puede servir para codificar y describir las respuestas de varias preguntas, por ejemplo, cuando hay varias con respuestas $\mathrm{Si} / \mathrm{No}$, codificadas como "1 = Si" y "2 = No". En otros casos, un tipo de rótulo puede servir para codificar las respuestas de una sola pregunta, por ejemplo, "1 = Masculino" y " $2=$ Femenino" para sexo, considerando un formulario donde no haya más preguntas que admitan estas respuestas. Siendo 1 y 2 los códigos, la diferencia se establece con el tipo de rótulo, así:

$\begin{array}{rrl}\text { Tipo de rótulo } & \text { Código } & \text { Rótulo } \\ 1 & -1 & \text { Total } \\ 1 & 1 & \text { Masculino } \\ 1 & 2 & \text { Femenino } \\ 2 & -1 & \text { "Total empresa" } \\ 2 & 1 & \text { Si } \\ 2 & 2 & \text { No }\end{array}$

Hemos agregado para cada tipo de rótulo una línea con el código -1 para incluir los datos del total del grupo estudiado. El valor -1 es arbitrario, pero conveniente para separar visualmente los diferentes tipos de rótulo. Como se verá más adelante, el programa agrega un tipo 0 (cero) con código 0 y rótulo en blanco para las variables numéricas.

Puede suceder que, por el diseño del formulario, se codifiquen respuestas iguales de manera diferente en varias preguntas. Por ejemplo, para la pregunta 5 se toma " $0=$ No" y " $1=$ Si". Éste sería otro tipo de rótulo y se incluiría como un tipo diferente del anterior. Por ejemplo:

$\begin{array}{rrl}\text { Tipo de rótulo } & \text { Código } & \text { Rótulo } \\ 1 & -1 & \text { Total } \\ 1 & 1 & \text { Masculino } \\ 1 & 2 & \text { Femenino } \\ 2 & -1 & \text { "Total empresa" } \\ 2 & 1 & \text { Si } \\ 2 & 2 & \text { No } \\ 3 & -1 & \text { "Total empresa" } \\ 3 & 0 & \text { No } \\ 3 & 1 & \text { Si }\end{array}$

Se debe tomar nota del uso de las comillas cuando el rótulo tiene más de una palabra. Si es de una sola, el uso de las comillas es opcional.

\subsection{Las variables}

Corresponden a algún campo del formulario o se definen a partir de transformaciones. Cada una debe tener un número que la identifica y que sirve como enlace 
para obtener los valores de las estimaciones que se incluirán en las tablas editadas. Si la variable es categórica, tendrá asociado un tipo de rótulo que describe las respuestas obtenidas. Si es numérica, se le asignará como tipo de rótulo el valor 0 (cero). De esta manera, para cada variable tendremos un número que la identifica, un tipo de rótulo asociado y un nombre que la describe. Por ejemplo:

$\begin{array}{ccl}\text { Número } & \text { Tipo de rótulo } & \text { Nombre } \\ 11 & 1 & \text { Sexo } \\ 21 & 2 & \text { "Ha salido del país" } \\ 24 & 3 & \text { "Tiene tarjeta Diners" } \\ 25 & 3 & \text { "Tiene tarjeta Visa" } \\ 26 & 3 & \text { "Tiene tarjeta MasterCard" } \\ 35 & 0 & \text { Edad }\end{array}$

Las respuestas de las preguntas 21 y 24 son "Si/No", pero se supone que en la 21 están codificadas como 1 y 2 , mientras que en la 24 lo están como 1 y 0 . La variable Edad no es categórica, entonces tiene asociado el tipo de rótulo 0 (cero). Obsérvese también el uso de las comillas en los nombres con más de una palabra.

\subsection{Los títulos de las tablas}

Cada tabla lleva un título que describe su contenido. La elaboración de un listado en un mismo lugar permite analizar la secuencia de los temas y eventualmente hacer una reorganización. Cada uno lleva un número para identificarlo, pero esa numeración la genera el procedimiento, como se verá más adelante. Como ejemplo:

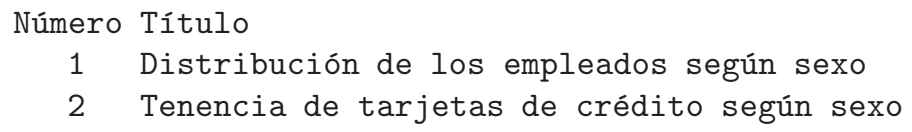

Es posible que algunos títulos no se utilicen. Esto no genera ningún problema, pero lo más razonable es incluir solo los que servirán para alguna de las tablas.

\subsection{Notas}

Las notas son opcionales, pero convenientes si se quiere agregar información que se considera útil para comprender la tabla o para dar a conocer la fuente de los datos. Se elabora un listado similar al de los títulos, sin numeración, pues el procedimiento la genera. Se debe crear al menos una nota ficticia así no se utilice:

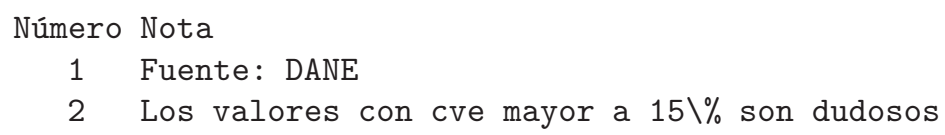




\subsection{Descripción de las filas de las tablas}

Como dijimos antes, consideramos tablas en las que cada fila corresponde a una variable de la cual se obtienen las estimaciones. Las variables incluidas en cada una deben tener un tema común que sirva para contextualizar su análisis. Operativamente lo llamaremos el encabezado de las filas de la tabla y su introducción es similar a la de los títulos. Por ejemplo:

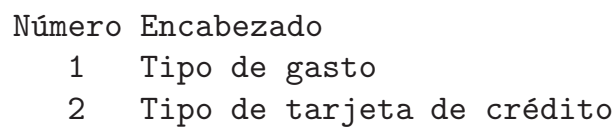

\subsection{Descripción de las columnas de las tablas}

En las columnas se clasifica la población en grupos determinados por una de las variables categóricas, que los muestristas acostumbran llamar dominios. El nombre de la variable utilizada describe este tipo de clasificación. No se elabora ninguna tabla, pero se toma nota del número del campo del formulario que va a cumplir este papel. Por ejemplo, si los grupos poblacionales están determinados por el sexo, esta es la variable que determinará los dominios o las clases donde se calculan las estimaciones. El número que se utilizó para esta variable es $11(\S 2.2)$ y forma parte de la información que se utiliza para definir una tabla, como se verá en la sección siguiente.

\subsection{Las tablas}

La reunión de las informaciones anteriores define una tabla particular que se identificará dentro del sistema con un número de tabla. Este número puede no coincidir con el que aparece en el informe, pues allí la numeración es automática. La tabla se define con la información siguiente:

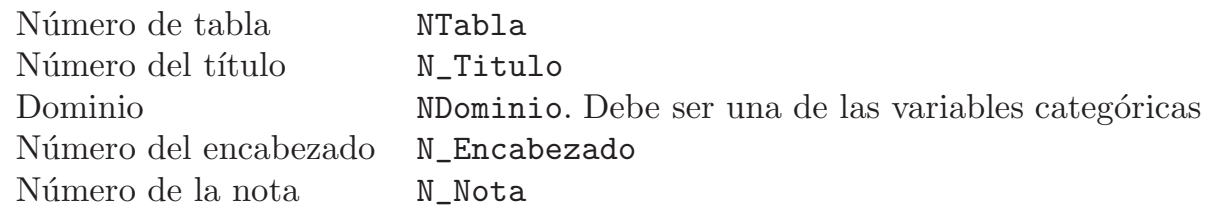

Ilustramos la definición de una tabla, identificada en el sistema con el número 21, con el título número 1 que corresponde al texto "Distribución de los empleados según sexo", con subgrupos poblacionales por sexo, cuyo código es 11 (§ 2.2), con filas que indican la tenencia de tarjetas de crédito Diners, Visa o Mastercard. Por ello, se utiliza el encabezado con código 2 (§ 2.5). Además, se agrega la nota sobre la fuente cuyo código es 1 ( $\S$ [2.4). Esta información se presenta de la siguiente manera:

2111121 
Cada línea define una tabla diferente. El conjunto de tablas preparadas para el informe está entonces compuesto por el conjunto de líneas en el arreglo anterior. LATEX se encargará de asignarles una numeración y etiquetas de la forma TablaXX, donde XX es un número asignado por el sistema para hacer referencia en el análisis.

\subsection{Las estimaciones}

Son los resultados de las rutinas de estimación aplicadas a los datos obtenidos con los formularios de recolección de información. Todo el trabajo de edición busca presentarlas de manera conveniente.

Una línea de archivo de estimaciones debe iniciar con el número de tabla ( $\$$ 2.7]), el número del campo o variable $(\S 2.2)$ que corresponda a las estimaciones presentadas y luego la estimación del grupo total, seguida de las de las subpoblaciones o dominios. Luego, en la misma línea, el coeficiente de variación de la estimación para el grupo total, seguida de las de los dominios. Por ejemplo, para la tenencia de tarjetas de crédito:

$\begin{array}{lllllllll}21 & 24 & 20.1 & 21.4 & 19.3 & 5 & 18.6 & 15.1\end{array}$

$\begin{array}{lllllllll}21 & 25 & 10.8 & 18.2 & 16.7 & 8 & 10.4 & 12.6\end{array}$

$\begin{array}{llllllll}21 & 26 & 12.7 & 23.0 & 17.2 & 3 & 8.9 & 11.8\end{array}$

\section{Procedimiento}

Active $\mathrm{R}$ y ejecute los programas del apéndice A para tener disponibles las funciones TbLatex y DataFr.

Abra el editor de programas (ArchivolNuevo script).

\subsection{Generación de los rótulos}

Construya una tabla de rótulos como se indicó en la sección 2.1] Reemplace en el modelo siguiente los datos por los suyos, sin dejar líneas en blanco salvo la última que indica el final de los datos. No olvide evitar en los textos el uso de símbolos que utiliza $\mathrm{R}$ como términos o símbolos claves, por ejemplo: \%, \.

$\begin{array}{crl}\text { Rótulo }=\operatorname{scan}(\text { what }= & \text { character }(0)) \\ 1 & -1 & \text { Total } \\ 1 & 1 & \text { Masculino } \\ 1 & 2 & \text { Femenino } \\ 2 & -1 & \text { "Total empresa" } \\ 2 & 1 & \text { Si } \\ 2 & 2 & \text { No } \\ 5 & -1 & \text { Total }\end{array}$

Comunicaciones en Estadística, junio 2009, Vol. 2, No. 1 


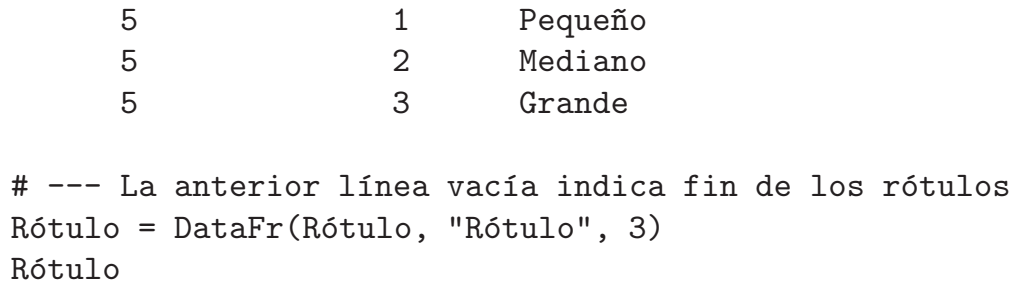

Las dos últimas líneas llaman la función DataFr para adecuar la estructura de los datos a la forma que necesita el programa. No las modifique.

\subsection{Información sobre las variables}

Igual que con los rótulos, ingrese sus datos como se indicó en la sección 2.2. Puede utilizar el siguiente modelo, reemplazando los datos por los suyos:

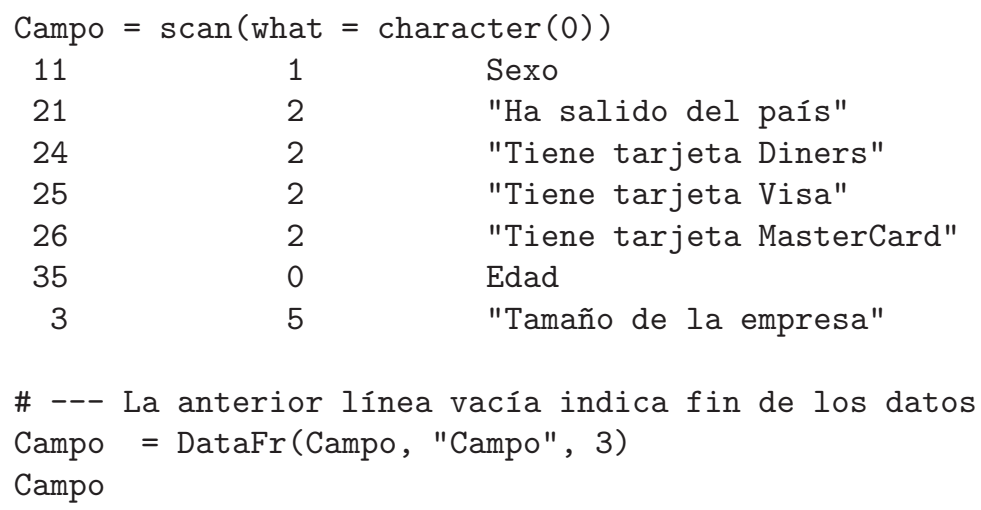

Como antes, las dos últimas líneas llaman la función DataFr para adecuar la estructura de los datos como la necesita el programa. No las modifique.

\subsection{Información sobre los títulos}

Para los títulos solo introduzca los textos respectivos, uno por fila, sin utilizar comillas. La numeración es automática, como verá cuando ejecute el código siguiente, luego de reemplazar los textos por los suyos:

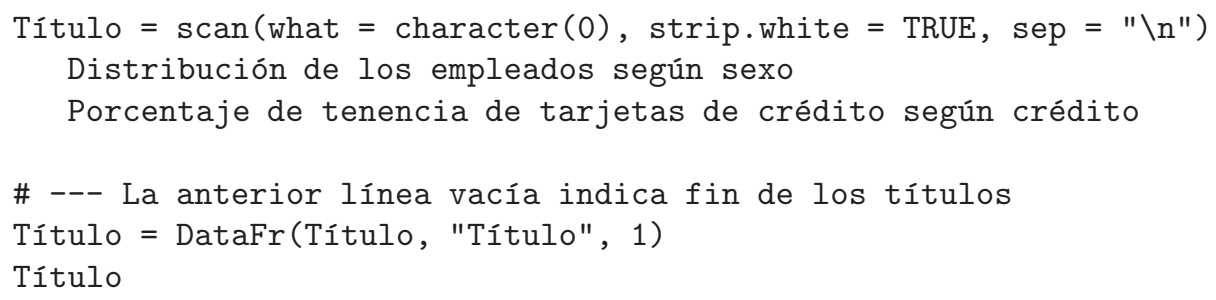




\subsection{Información sobre las notas de las tablas}

Igual que los títulos, los textos de las notas se introducen sin numeración y sin comillas, uno por fila. La numeración es automática. En caso de necesitar utilizar un símbolo especial de $\mathrm{R}$ dentro de su texto, debe precederlo de $\backslash$, como se muestra en la segunda nota. No hacerlo implicará problemas durante la compilación. Esto aplica para todos los textos, incluidos los anteriores.

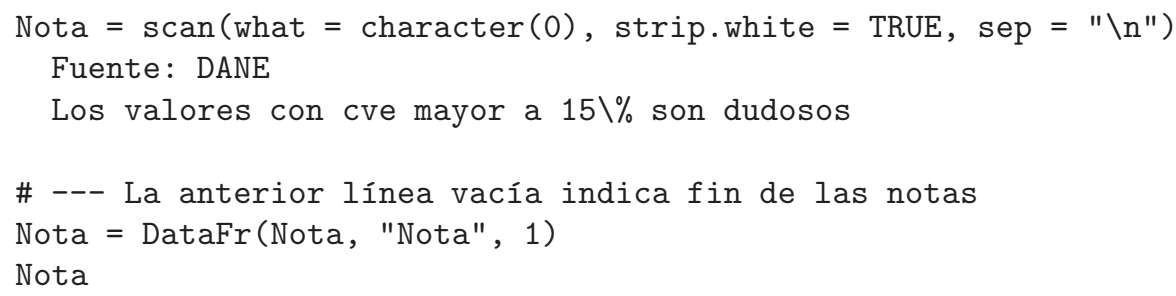

\subsection{Descriptores de las filas de las tablas}

Son los que hemos llamado encabezados de filas y sirven para mostrar el contexto que unifica las diferentes filas de la tabla. Se introducen igual que las notas o que los títulos, sin numeración, pues el programa la genera automáticamente.

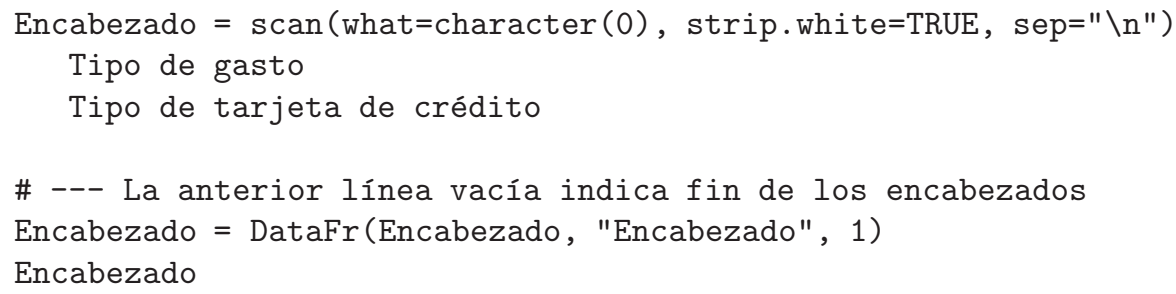

\subsection{Construcción de las tablas}

Como se vio en la sección 2.7 para preparar una tabla, se le asigna un número, un título, una variable que defina los dominios, un encabezado de filas y una nota. Si no hay nota para la tabla, se escribe 0 (cero).

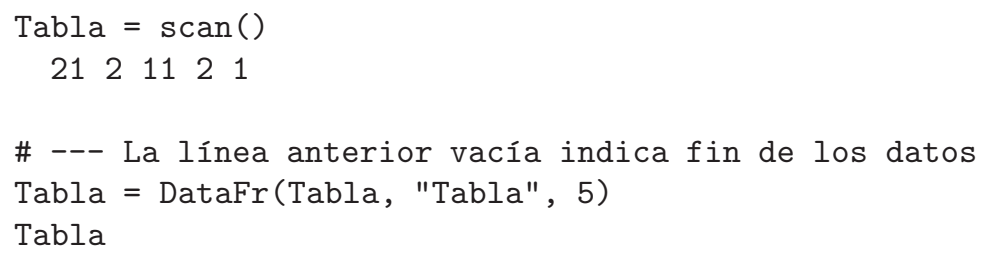




\subsection{Adecuación de las estimaciones}

En este paso del proceso se utiliza el número de la tabla creada en el paso anterior para marcar cada una de sus filas. Si $k$ es el número de categorías de la variable que define los dominios, el número de columnas de este archivo es igual a $2 \times(k+2)$. Por ejemplo, si los dominios corresponden a los grupos según sexo, $k=2$ y las columnas de este archivo serán $8=2 \times(2+2)$. Este es el valor que debe introducir para el último argumento de la función.

\begin{tabular}{|c|c|c|c|c|c|c|}
\hline \multicolumn{7}{|c|}{ Estimación $=$ scan ()} \\
\hline 21 & 24 & 13.4 & 14.8 & 12.3 & 16.7 & 2.11 .0 \\
\hline 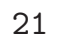 & 25 & 3.2 & 3.3 & 4.8 & 5.7 & 4.12 .1 \\
\hline 21 & 26 & 11.2 & 10.7 & 11.1 & 2.5 & 2.93 .7 \\
\hline
\end{tabular}

\# --- La anterior línea vacía indica fin de los datos

Estimación = DataFr(Estimación, "Estimación", 8)

Estimación

\section{Generación del código fuente $\mathrm{AT}_{\mathrm{E}} \mathrm{X}$}

Reemplace la carpeta que se muestra entre comillas por la que desea utilizar para guardar el informe (no incluya el nombre del archivo). Ejecute luego las instrucciones siguientes:

Carpeta $=$ "c:/Escritos/RevistaUSTA/TablasLatex"

setwd (Carpeta)

TbLatex(Carpeta, Tabla, Estimación, Campo, Rótulo, Título, Nota, 0)

Si sus datos fueron introducidos correctamente, recibirá como respuesta la carpeta utilizada y el nombre del archivo donde quedó el documento fuente $\mathrm{AT}_{\mathrm{E}} \mathrm{X}$.

El nombre del archivo generado es Tablas_0.tex, listo para compilar o, si lo prefiere, para agregar los comentarios resultantes de su análisis. Después de cada tabla encontrará un par de líneas que le indican donde hacerlo. Cada tabla se puede referenciar con la etiqueta \ref $\{\mathrm{TablaXX}\}$, donde XX es el número asignado por el programa. Con los datos anteriores, el resultado se encuentra en la tabla 2

Antes de cerrar la sesión de R, guarde el archivo de instrucciones, preferiblemente en la misma carpeta, para tener disponible el código de generación del informe si llega a necesitarlo.

El último argumento de la función TbLatex sirve para generar las tablas de manera vertical (0) o apaisada, si cambia 0 por 1. 
Tabla 2: Porcentaje de tenencia de tarjetas de crédito según sexo

\begin{tabular}{|c|c|c|c|}
\hline \multirow[b]{2}{*}{$\begin{array}{l}\text { Tipo de tarjeta de } \\
\text { crédito }\end{array}$} & \multirow[b]{2}{*}{ Total } & \multicolumn{2}{|c|}{ Sexo } \\
\hline & & Masculino & Femenino \\
\hline Tiene tarjeta Diners & 13.4 & 14.8 & 12.3 \\
\hline (cve\%) & 16.7 & 2.1 & 1 \\
\hline Tiene tarjeta Visa & 3.2 & 3.3 & 4.8 \\
\hline (cve\%) & 5.7 & 4.1 & 2.1 \\
\hline $\begin{array}{l}\text { Tiene tarjeta Master- } \\
\text { Card }\end{array}$ & 11.2 & 10.7 & 11.1 \\
\hline (cve \%) & 2.5 & 2.9 & 3.7 \\
\hline
\end{tabular}

Fuente: DANE

\section{Ejemplo}

La siguiente es la secuencia de instrucciones que se elabora para generar las tablas 3 y 4 con datos ficticios.

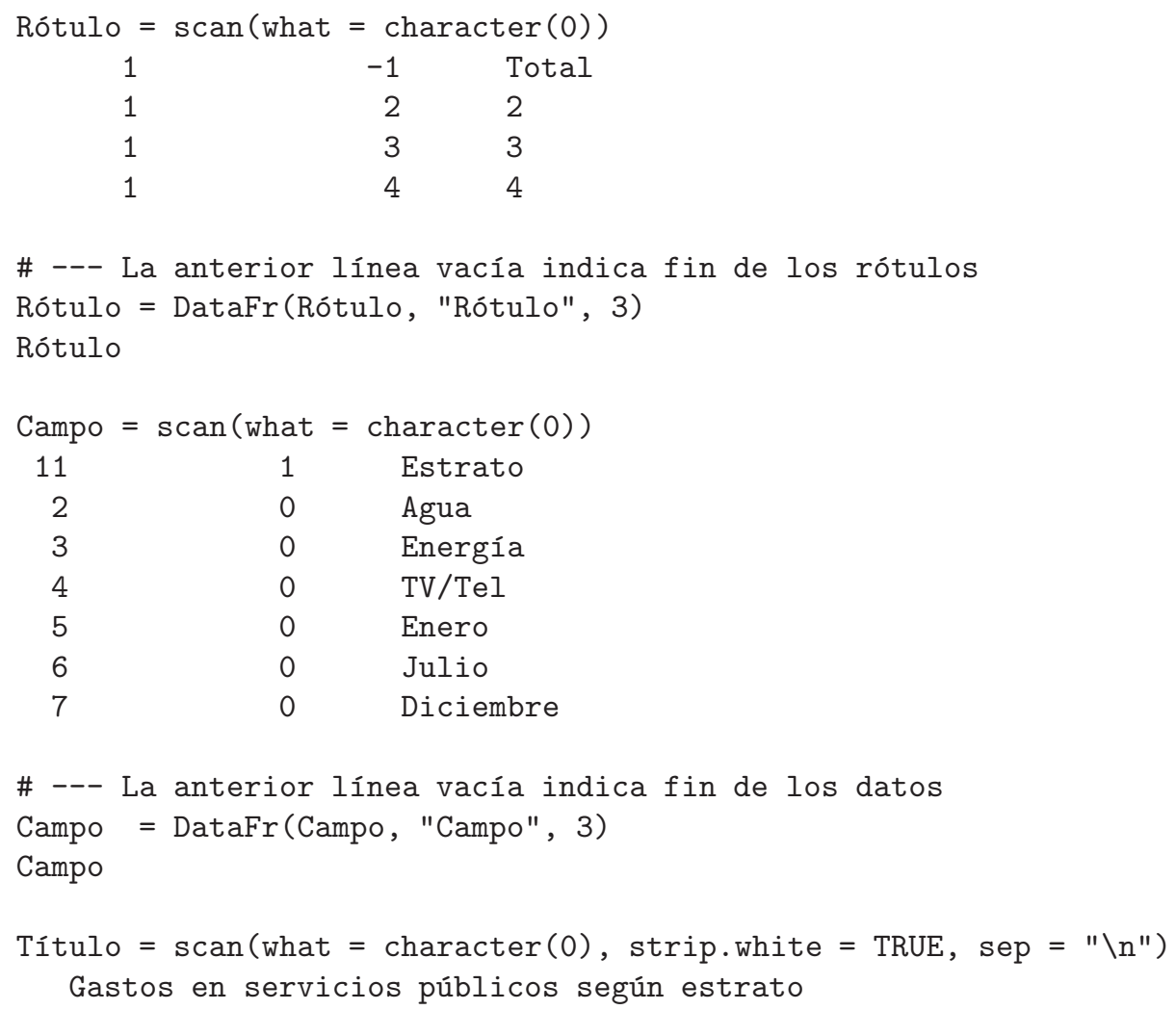




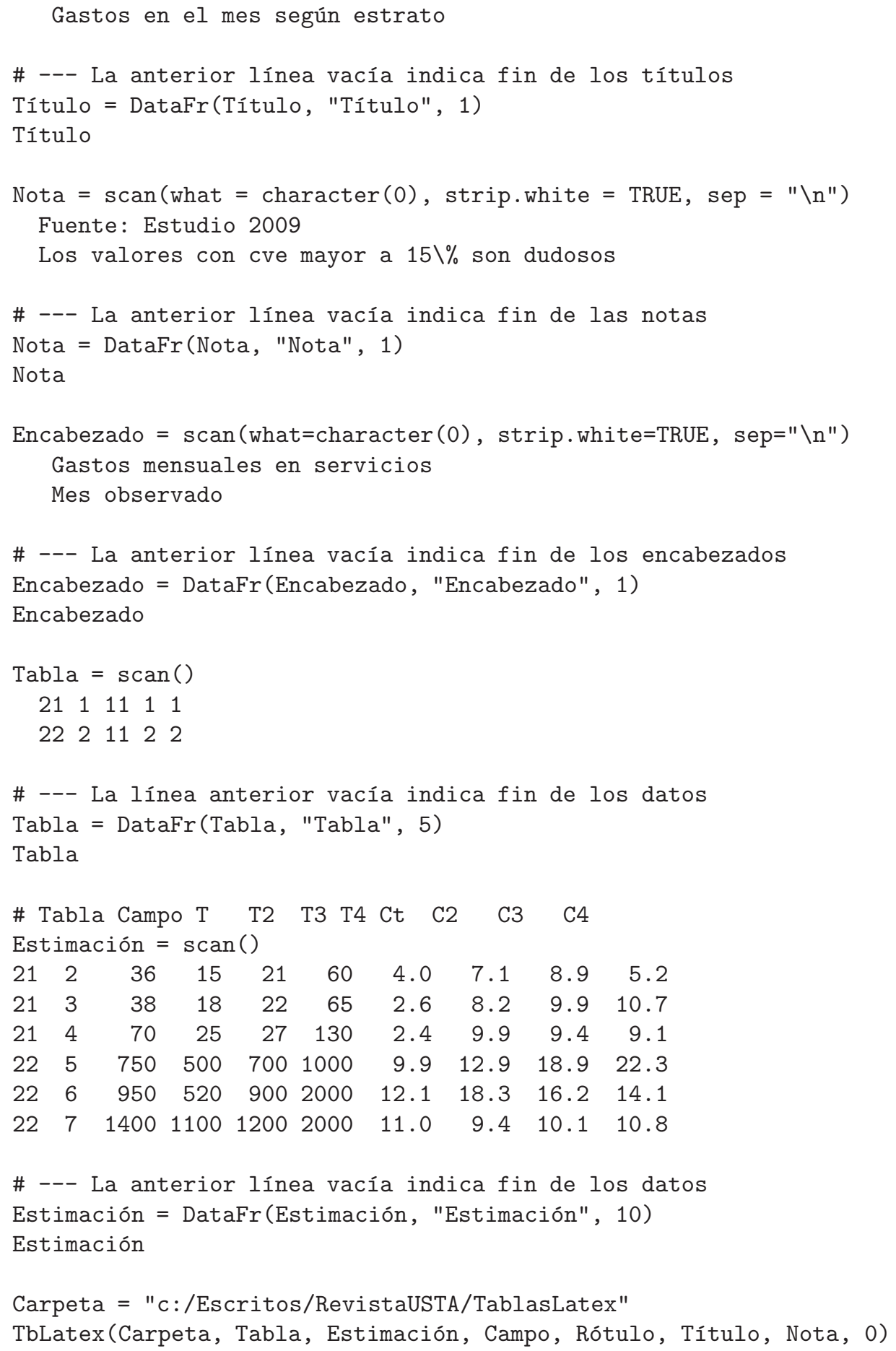


Tabla 3: Gastos en servicios públicos según estrato

\begin{tabular}{|c|c|c|c|c|c|}
\hline \multirow{2}{*}{\multicolumn{2}{|c|}{$\begin{array}{l}\text { Gastos mensuales en } \\
\text { servicios }\end{array}$}} & \multirow[b]{2}{*}{ Total } & \multicolumn{3}{|c|}{ Estrato } \\
\hline & & & 2 & 3 & 4 \\
\hline \multirow[t]{2}{*}{ Agua } & & 36 & 15 & 21 & 60 \\
\hline & (cve\%) & 4 & 7.1 & 8.9 & 5.2 \\
\hline \multirow[t]{2}{*}{ Energía } & & 38 & 18 & 22 & 65 \\
\hline & (cve\%) & 2.6 & 8.2 & 9.9 & 10.7 \\
\hline \multirow[t]{2}{*}{$\mathrm{TV} / \mathrm{Tel}$} & & 70 & 25 & 27 & 130 \\
\hline & (cve\%) & 2.4 & 9.9 & 9.4 & 9.1 \\
\hline
\end{tabular}

Fuente: Estudio 2009

Tabla 4: Gastos en el mes según estrato

\begin{tabular}{|c|c|c|c|c|c|}
\hline \multirow{2}{*}{\multicolumn{2}{|c|}{ Mes observado }} & \multirow[b]{2}{*}{ Total } & \multicolumn{3}{|c|}{ Estrato } \\
\hline & & & 2 & 3 & 4 \\
\hline \multirow[t]{2}{*}{ Enero } & & 750 & 500 & 700 & 1000 \\
\hline & (cve\%) & 9.9 & 12.9 & 18.9 & 22.3 \\
\hline \multirow[t]{2}{*}{ Julio } & & 950 & 520 & 900 & 2000 \\
\hline & (cve\%) & 12.1 & 18.3 & 16.2 & 14.1 \\
\hline \multirow[t]{2}{*}{ Diciembre } & & 1400 & 1100 & 1200 & 2000 \\
\hline & (cve\%) & 11 & 9.4 & 10.1 & 10.8 \\
\hline
\end{tabular}

Los valores con cve mayor a $15 \%$ son dudosos

\section{Conclusiones}

El trabajo de edición de las tablas de estimaciones en un estudio de encuestas puede simplificarse de manera importante con el uso de programas como el presentado en este artículo. Además, la preparación puede iniciarse desde el momento en que los investigadores tienen definido el conjunto de tablas que utilizarán para su análisis. Así, cuando llegan los datos, el conjunto de tablas se genera muy rápidamente en su presentación final y el analista puede dedicarse a incorporar los comentarios sin el riesgo de recibir tablas editadas manualmente que con mucha probabilidad contienen errores de transcripción.

\section{Referencias}

Bautista, L. (1998), Diseños de muestreo estadístico, Universidad Nacional de Colombia, Bogotá.

Comunicaciones en Estadística, junio 2009, Vol. 2, No. 1 
Ortiz Pinilla, J. E. (2009), Simulación y métodos estadísticos, Editorial Universidad Santo Tomás, Bogotá.

Ortiz Pinilla, J. E. \& Bautista, L. A. (2006), 'Tablas de encuestas en LATEX', Revista Colombiana de Estadística 29(2), 60-67.

R Development Core Team (2009), R: A Language and Environment for Statistical Computing, R Foundation for Statistical Computing, Vienna, Austria. ISBN 3-900051-07-0.

*http://www.R-project.org

Särndal, K. E., Swensson, B. \& Wrettman, J. (1992), Model Assisted Survey Sampling, Springer, New York.

\section{A. Programas}

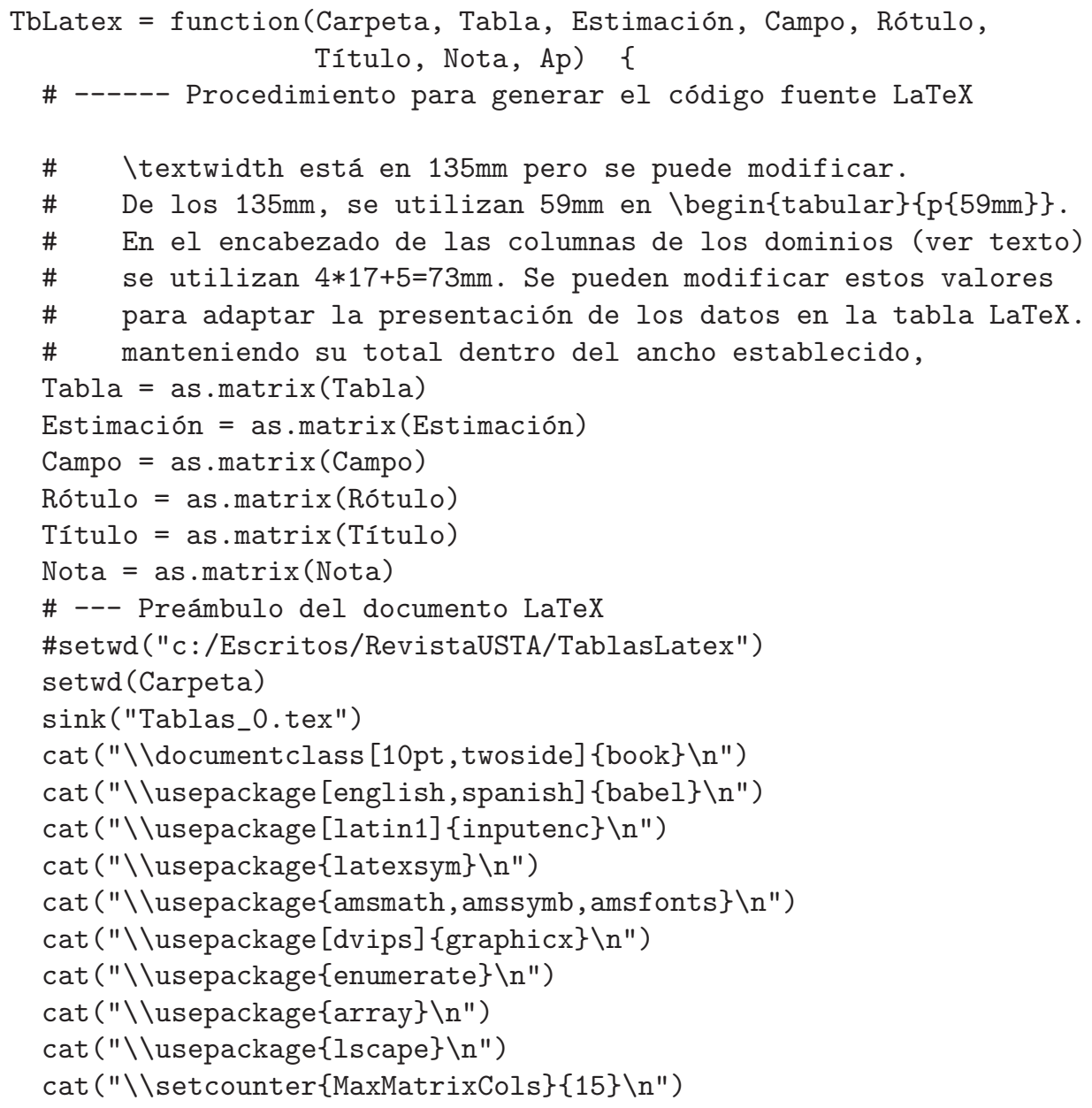




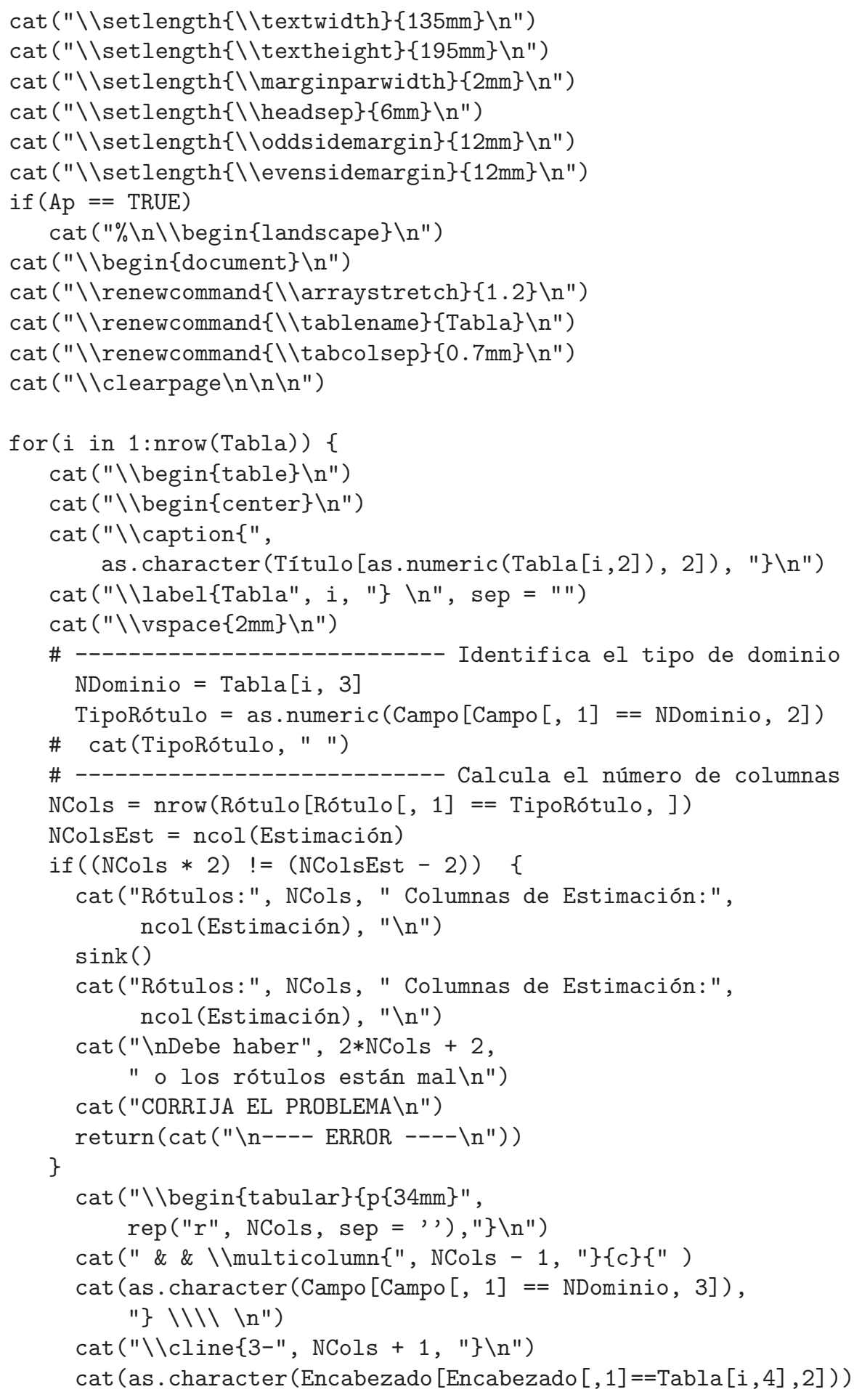




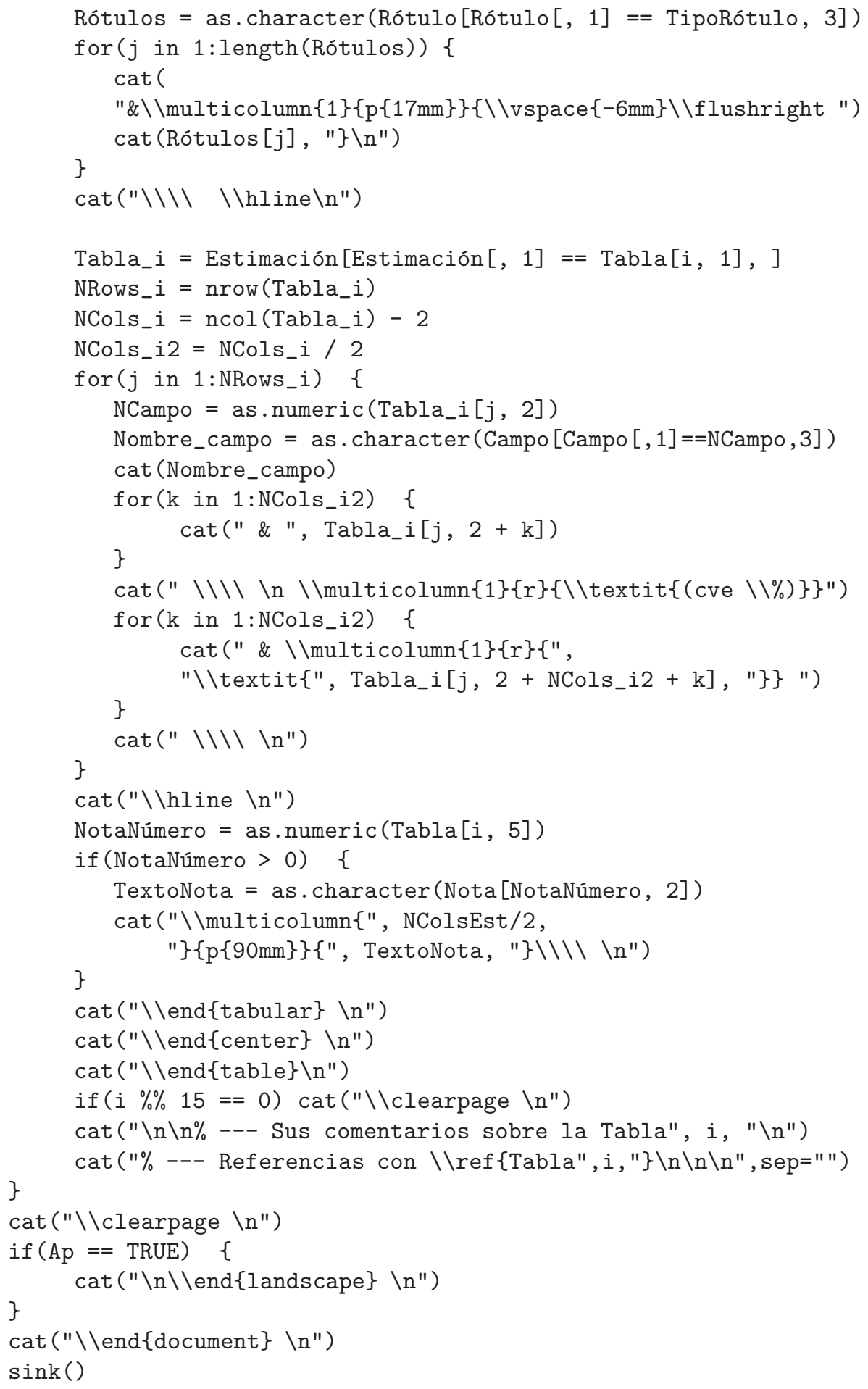




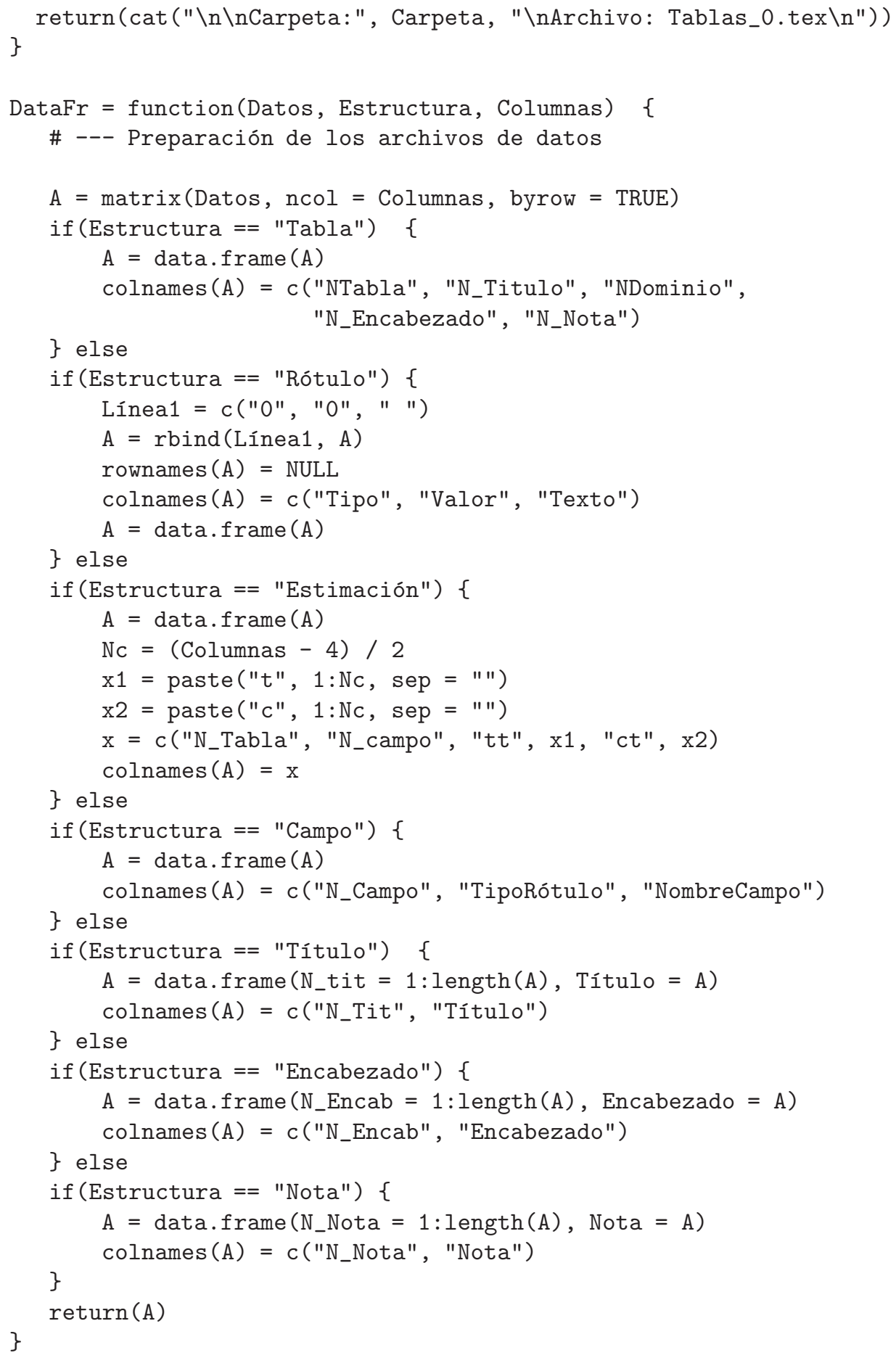

